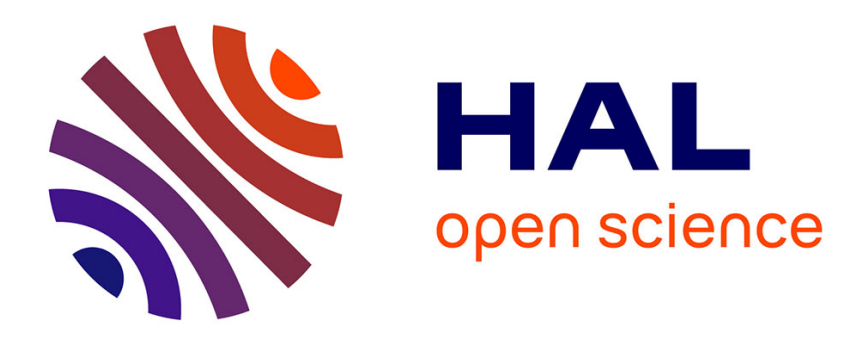

\title{
Filtration of ultrafine metallic particles in industry
}

\author{
Denis Bémer, Yves Morele, Roland Régnier
}

\section{To cite this version:}

Denis Bémer, Yves Morele, Roland Régnier. Filtration of ultrafine metallic particles in industry.

Environmental Technology, 2015, 36 (18), 10.1080/09593330.2015.1028471 . hal-01214371

\section{HAL Id: hal-01214371 \\ https://hal.science/hal-01214371}

Submitted on 12 Oct 2015

HAL is a multi-disciplinary open access archive for the deposit and dissemination of scientific research documents, whether they are published or not. The documents may come from teaching and research institutions in France or abroad, or from public or private research centers.
L'archive ouverte pluridisciplinaire HAL, est destinée au dépôt et à la diffusion de documents scientifiques de niveau recherche, publiés ou non, émanant des établissements d'enseignement et de recherche français ou étrangers, des laboratoires publics ou privés. 


\title{
Filtration of ultrafine metallic particles in industry
}

\author{
D. Bémer ${ }^{*}$ Y. Morele, Régnier R. \\ Institut National de Recherche et Sécurité, rue du Morvan, 54519 Vandoeuvre \\ (*) Email : denis.bemer@inrs.fr, Tel : (33)383 5020 00, fax : (33)383 805184
}

\begin{abstract}
Thermal metal spraying, metal cutting and arc welding processes generate large quantities of ultrafine particles that cause the irreversible clogging of industrial filters. The aim of the study performed was to identify the causes of the clogging of cartridge filters and investigate other paths for cleaning them. This study required the development of a test bench capable of reproducing a thermal spraying process to test the performances of different filtration techniques. This test instrument first, permitted the precise characterisation of the aerosol generated by the process and, second, defined the clogging and cleaning conditions for filters. Several parameters were tested: the type of filter, on-line and off-line cleaning, pre-coating, cleaning by jets of high speed compressed air via a probe.
\end{abstract}

Keywords : ultrafine particles; thermal spraying; filtration; dust collector

\section{Introduction}

Many industrial processes give rise to the formation of aerosols composed of ultrafine particles (UFP, particle diameter $<100 \mathrm{~nm}$ ) which, in addition to their specific toxicity due to their size and chemical composition, lead to the clogging of the industrial filters used to eliminate them. Clogged filters result in increased resistance to the flow of air through the filter, leading to a deterioration of the performances of the ventilation installed (booth, suction table, etc.).

The primary particles thus formed by condensation of the metallic vapour agglomerate into bigger particles, mainly by the action of the Brownian diffusion mechanism, up to 300-500 nm [1]. Particles greater than $500 \mathrm{~nm}$ are eliminated by deposition on the walls of the different structures (ducts, ventilated booth, etc.) by sedimentation or inertial deposition. Three hundred nanometers corresponds precisely to the MPPS (Most Penetrating Particle Size) i.e. the particle size the most difficult to be retained by media filters, electrofilters, wet scrubbers, cyclones, etc. [2,3] Actually, filtration using fibrous media presents the best compromise performances/costs and is, for this reason, the separating technique the most widely used in industry for particle removal.

Thermal metal spraying (flame, electric arc, etc.), metal cutting (arc/air process) and arc welding processes generate large quantities of UFP (concentration in number $>10^{8} / \mathrm{cm}^{3}$, mass concentration $>100 \mathrm{mg} \cdot \mathrm{m}^{-3}$, [4]), that are often highly toxic, and the cause of irreversible clogging of the filters used in dust extraction installations. The size of the primary particles composing these aerosols and their aggregates is sometimes very small $(9 \mathrm{~nm}$ for the primary particles of thermal spraying, $70-100 \mathrm{~nm}$ for the aggregates), due to the almost instantaneous dilution caused by the injection of compressed air at the point of metal fusion. The particles generated by arc welding, for example, which can also be considered as ultrafine, present larger primary particles $(40-90 \mathrm{~nm})$ [5], due to the low dilution at the point of 
fusion. However, the larger the specific surface of the particles (ratio of the surface developed by the particles to the volume), the higher the clogging capacity of the layer of particles [6].

The most pollutant operations like thermal metal spraying are performed in ventilated booths. The air contaminated by the particles is directed to the dust extraction installation whose air flow is determined by the section of the booth to be ventilated, i.e. $1400 \mathrm{~m}^{3} / \mathrm{h} / \mathrm{m}^{2}$ [7], which leads to high flow rates, generally between 20000 and $25000 \mathrm{~m}^{3} / \mathrm{h}$.

Filtration using pleated filter cartridges is by far the most widely used air treatment technique in industry due to the large filter surface presented by the filters. However the clogging of the filters subject to metallic fumes is currently a source of problems. Indeed, observations in the field have shown that in many cases, clogged filters (Figure 1) are either maintained in the dust collector, in which case the ventilation flows are no longer ensured, resulting in poorer indoor air quality, or simply extracted, leading to the direct discharge of pollutants into the external environment.

The evolution of the filter pressure drop over filtration/cleaning cycles depends on many parameters [8]: the nature of the medium (elasticity, fibre diameter, etc.), the filter (shape, filtration surface, etc.), the pressure of the compressed air used for cleaning, etc. At present, the manufacturers of industrial dust collectors used for metallic UFP filtration, often have no solution other than increase the filtration surfaces in order to decrease filtration velocity and thus limiting further pressure drop and the frequency of filter replacement. Furthermore, increasing filtration surfaces leads to increased investment and operating costs.

The aim of the study performed by the INRS was to identify the causes of cartridge filter clogging and investigate other paths for cleaning filters. This study required developing a test bench capable of reproducing a thermal metal spraying operation by electric arc to test the performances of different filtration techniques. This test resource first permitted the precise characterisation of the aerosol generated with respect to the granulometry and morphology of the materials. Second, the clogging and above all the cleaning conditions of the filters, that is to say their capacity to eliminate the layer of metallic particles causing the increased pressure drop, were studied.

\section{Methodology}

The aerosol was generated by a thermal metal spraying process using a Margarido® M25 electric arc gun fed by wires made of $\mathrm{Zn} / \mathrm{Al}$ (85/15\%). The molten metal is sprayed by compressed air onto a rotating metal cylinder. The slag from the melting process is recovered in a tank located in the lower part of the chamber. The fumes and fine particles are extracted by a fan located at the end of the main channel $(200 \mathrm{~mm}$ in diameter) and directed to a cyclone that eliminates the micronic fraction of the particles but conserves the ultrafine fraction corresponding to the fumes. A fraction of the flow is thus directed to the filtration system studied. The remaining fumes are conveyed to the main dust separator (Figure 2).

The aerosol produced was characterised using different analysers. A Scanning Mobility Particle Sizer SMPS (Grimm 5.403), after dilution at 1/100 (Palas, VKL 100), was used to measure the number particle size distribution. The mass concentration was determined by 
collection on a filter (filter: GLA $5000 \mathrm{D} 47 \mathrm{~mm}$ ) and by gravimetry. A Tapered Element Oscillating Microbalance TEOM analyser (1400a, R\&P) was also used to monitor the evolution of the downstream mass concentration through time

The variation in pressure drop $(\triangle P)$ of a flat filter (GLA 5000) was measured during clogging. This measure permitted determining the specific resistance coefficient of the particle layer $\mathrm{k}_{2}$, according to Darcy's law: $\Delta \mathrm{P}=\mathrm{k}_{2} \mu \cdot \mathrm{U}_{\mathrm{f}} \cdot \mathrm{M}_{\mathrm{s}}$

With $\mu$, the dynamic viscosity of the gas, $\mathrm{U}_{\mathrm{f}}$, the filtration velocity and $\mathrm{M}_{\mathrm{s}}$ the mass of particles per unit of filter surface. Knowing the coefficient $k_{2}$ and the filtration conditions (air flow rate, particle mass concentration), it is theoretically possible to determine the kinetics of increasing $\triangle \mathrm{P}$ during clogging. Eq.1 also shows that for a given particulate pollutant, the only way to limit the $\triangle P$ increase is to reduce the filtration velocity as far as possible.

Two types of pleated cartridges were studied. They are manufactured using the same media (cellulose covered by a PTFE membrane) but present different filter surface areas and fold widths (Table 1).

Table 1. Characteristics of pleated cartridges.

\begin{tabular}{|c|c|c|}
\hline Medium & Cartridge $A$ & Cartridge $B$ \\
\hline Filter surface, $\mathrm{S}\left(\mathrm{m}^{2}\right)$ & 17.7 & 8.5 \\
\hline Fold height, $\mathrm{H}(\mathrm{mm})$ & 38.1 & 38.1 \\
\hline Fold width, L $(\mathrm{mm})$ & 2.84 & 6.03 \\
\hline Ratio H/L & 13.4 & 6.3 \\
\hline
\end{tabular}

The cartridge was placed horizontally in a closed chamber supplied by air recovered from the test bench. Cleaning was ensured by injecting compressed air in reverse direction flow via a nozzle placed at the inlet of the cartridge. The pressure of the compressed air was 4 bar and the flow rate of the test air was set at $340 \mathrm{~m}^{3} \cdot \mathrm{h}^{-1}$, resulting in filtration velocities for cartridges $A$ and $B$ of $0.55 \mathrm{~cm} / \mathrm{s}$ and $1.1 \mathrm{~cm} / \mathrm{s}$, respectively.

A second cleaning device, developed by the INRS, was tested. It comprised a probe supplied with compressed air placed inside the cartridge. The compressed air (at a pressure of 7 bar), discharged through several nozzles, swept the entire internal surface of the cartridge by rotating about its own axis (Figure 3 ). The cartridge was completely unclogged by four rotations of the probe.

A dust separator composed of four panel type filters, made of a quite rigid polyester covered with a PTFE membrane and placed vertically, was also tested. The filtration surface provided by the filters was $14 \mathrm{~m}^{2}$. Cleaning was performed by injecting compressed air in reverse direction flow. 
The pressure of the compressed air for cleaning was 6 bar and the air flow rate was set at $320 \mathrm{~m}^{3} \cdot \mathrm{h}^{-1}$ (filtration velocity $0.6 \mathrm{~cm} \cdot \mathrm{s}^{-1}$ ).

Pre-coating tests were performed in the cases of cartridges A and B. This operation consisted in depositing a layer of quite coarse and non toxic particles beforehand on the filter medium, the layer acting like a filter for the fume particles [9]. This layer above all presents the advantage of being easy to dislodge. Limestone dust (mass median diameter $3.6 \mu \mathrm{m}$ ) was used for this purpose. A mass of $200 \mathrm{~g}$ was injected over a period of ten minutes into the upstream air circuit (Figure 1) before starting metallic fume filtration. The mass per unit area deposited on filter can be determined from the filter surface of the filters. Tests were also performed with coarser dolomite dust (mass median diameter $9.5 \mu \mathrm{m}$ ) and a higher generated mass of $350 \mathrm{~g}$.

The conditions for initiating cleaning were also studied: cleaning was initiated with the air flow rate maintained (V-on) or not (V-off), and with the generation of metallic fumes activated (G-on) or not (G-off). There was obviously only one configuration in V-off mode, since the thermal spraying operation was, of course, stopped in the absence of ventilation.

\section{Results and discussion}

\subsection{Characteristics of metallic fume particles (downstream of the cyclone).}

Figure 4 shows the particle size distribution in number downstream of the cyclone, measured using the SMPS analyser, after dilution at $1 / 100$. The mean electrical mobility diameter in number is from 70 to $80 \mathrm{~nm}$ for a concentration of $2.10^{8} \mathrm{~cm}^{-3}$, the mass mean diameter is from 100 and $150 \mu \mathrm{m}$ for a concentration of $85 \mathrm{mg} \cdot \mathrm{m}^{-3}$. Figure 5 shows a photograph of particles taken by transmission electron microscopy. The mean diameter of the primary particles is $9 \mathrm{~nm}$. The porosity of the strongly aggregated particles causes their density to be much lower than the density of the constitutive material (metal). For example, in the case of $\mathrm{Zn} / \mathrm{Al}$ particles, the apparent mean density is $1 \mathrm{~g} / \mathrm{cm}^{3}$ for a material density equal to 5.74 $\mathrm{g} / \mathrm{cm}^{3}$. The apparent density of $\mathrm{Zn} / \mathrm{Al}$ particles was determined using a Differential Mobility Analyser DMA (Grimm 5.403) and an Aerosol Particle Mass analyser APM (Kanomax, 3601) in series [10]

\subsection{Coefficient $k_{2}$}

The measures performed at a flow rate of $4 \mathrm{l} / \mathrm{min}$, give a value of coefficient $\mathrm{k}_{2}=8.10^{11} \mathrm{~m} / \mathrm{kg}$ $\pm 1.6 .10^{11} \mathrm{~m} / \mathrm{kg}$ according to the operating conditions. These values appear to be quite stable for most thermal spraying processes, providing that the aerosol fraction $>1 \mu \mathrm{m}$ is eliminated. Values of the same magnitude have been obtained with metallic fumes generated by air arc cutting. This value of $k_{2}$ leads to a pressure drop increase kinetics of $145 \mathrm{~Pa} /(\mathrm{g} / \mathrm{m} 2 /(\mathrm{cm} / \mathrm{s})$. Applied to the case of cartridge $B$, using a concentration of $85 \mathrm{mg} / \mathrm{m}^{3}$, the $\triangle P$ increase would therefore be $560 \mathrm{~Pa} / \mathrm{h}$.

\subsection{Filter performances}

The filtration flow rate and pressure drop were recorded continuously. The particle mass concentration was also measured upstream and downstream of the filter. Cleaning was initiated when the pressure drop reached a predefined limit. Three configurations were 
tested: V-on / G-on, V-on / G-off and V-off, with and without pre-coating. Pre-coating was not studied in the case of the panel filter.

\subsection{Cartridge filter}

Figure 6 shows the almost total lack of efficiency of cleaning on cartridge $B$ whether done in $\mathrm{V}$-on mode or V-off mode. During cleaning in V-off mode, the reduction of pressure drop is more marked, but the increase following the resumption of filtration is also very fast, so that the $\triangle P$ value returns to its value before cleaning almost immediately. Regarding cartridge $A$ which have a larger filtration surface, the same phenomenon was observed, though it was simply slower. Note that the increase of $\triangle P$ of the cartridge corresponds to the data obtained for the flat filter (coefficient $k_{2}$ ). The downstream mass concentrations varied from $2.5 \mathrm{mg} / \mathrm{m}^{3}$ at the start of the cycle (new or completely cleaned filter) to $0.15 \mathrm{mg} / \mathrm{m}^{3}$ at the end of the cycle. Note that this low value of the mass concentration $\left(0.15 \mathrm{mg} / \mathrm{m}^{3}\right)$ corresponds to a relatively high number concentration of $4.10^{5}$ particles $/ \mathrm{cm}^{3}$, compared for instance to the IFA (2009) limit value for oxide metallic UFP of $2.10^{4}$ to $4.10^{4}$ particles $/ \mathrm{cm}^{3}$ [11].

Cleaning using the compressed air probe was tested on cartridge B in V-off and V-on modes. The results (not shown) show complete cleaning of the cartridge with the total elimination of the cake of particles. After cleaning, the pressure drop returned to its initial value and the $\triangle P$ at the resumption of filtration was similar to that of a new cartridge. Continuing ventilation during the cleaning operation ( $\mathrm{V}$-on) did not appear to have an influence on the device's capacity to eliminate the layer of particles.

Following the pre-coating of cartridge B with limestone dust of particle size $3.6 \mu \mathrm{m}$, cleaning was performed when the ventilation was stopped (Figure 7, V-off). At the end of cleaning, the pressure drop of the cartridge returned to values close to the initial $\triangle P$, but the kinetics of increase was higher than that of a new cartridge, indicating partial cleaning. However, in Von / G-on mode cleaning was totally inefficient (similar results were obtained with cartridge A). With dolomite dust of particle size $9.5 \mu \mathrm{m}$, partial cleaning was nonetheless obtained in $V$-on mode, leading to a residual $\triangle P$ (after cleaning) higher than the initial $\triangle P$. This can be explained by the lower adhesion forces developed by this layer of particles, which were larger and therefore easier to detach.

Tests were also performed, still using pre-coating with dust of particle size $9.5 \mu \mathrm{m}$, in V-on mode, but this time stopping the thermal spraying operation several seconds before initiating the cleaning (G-off). In this configuration, the cleaning appeared to be more efficient and the pressure drop returned to the evolution described in Figure 7. The almost instantaneous filling of the cleaned surfaces by the fine metallic particles may explain the inefficiency of cleaning in V-on / G-on mode.

\subsection{Panel filters}

Figure 8 shows the evolution of the $\triangle P$ of the panel filter over several clogging/cleaning cycles in V-off mode. This time, the cleaning allowed eliminating a sufficient fraction of the filter cake to lower the $\triangle P$ durably. Cake detachment occurs by a process of "patchy cleaning", where some areas of cake are completely removed and others not at all (Figure 9) 
[12]. The small reductions (a hundred $\mathrm{Pa}$ ) observed for the $\triangle \mathrm{P}$ were caused by interruptions of the tests from one day to another. The test was interrupted for a much longer period of several weeks (indicated by the arrow in Figure 8). The kinetics of increasing $\triangle P$ on resuming filtration was therefore much slower. Then the system returned to the behaviour exhibited before the long interruption. The average efficiency of the filter determined over a clogging/cleaning cycle, based on the upstream/downstream particle mass concentrations, was $99.3 \%$ with a mean downstream concentration of $0.6 \mathrm{mg} / \mathrm{m}^{3}$. During the filter clogging, the formation of the layer of particles on the surface of the medium contributes to increasing the filtration efficiency. Thus the downstream concentration, measured with a TEOM analyser, regularly decreases during clogging as shown in Figure 10. A quite high concentration was recorded at the beginning of the cycle $\left(\approx 1.8 \mathrm{mg} / \mathrm{m}^{3}\right)$.

Contrary to the cartridge filter, the cleaning performances of the panel filter did not appear to be affected by maintaining ventilation and generation (V-on / G-on). With this mode of operation, cleaning made it possible to obtain a $\triangle P$ very close to the initial value and the kinetics of increased pressure drop when re-starting filtration was similar to that of a new filter. But the overly short tests performed, did not allow reaching a definitive conclusion. Nonetheless, it appears that the slackness conferred by the panel shape of the filter contributes to the improved cleaning observed. This phenomenon was highlighted by the works of Dennis [13] in a study in which he effectively showed that optimal cleaning of the layer of particles is obtained for slackness filters with very low intrinsic elasticity. This slackness of the medium provides it with a considerable freedom of movement during pressure cleaning pulse, generating considerable detachment forces during the deceleration phase of the medium, due to its low elasticity.

\section{Conclusion}

Observations in the field showing that dust collectors with pleated cartridge filters were irreversibly clogged, were confirmed by our laboratory results. Pulse-jet cleaning of these cartridges by compressed air injected in reverse direction flow appeared to be very inefficient in both on-line (with ventilation on) and off-line (ventilation off) modes. But this solution can nevertheless be retained if the user agrees to frequently change the cartridges, which will result in additional costs.

Use of the pre-coating technique permitted cleaning these cartridges, but only in off-line mode. In on-line mode, once again using pre-coating, cleaning could nonetheless be performed after stopping the thermal spraying operation. However, this solution should be tested on an industrial installation operating over a longer period. But one disadvantage of the pre-coating solution is that it generates large quantities of waste, made of the pre-coating powder contaminated but the metallic UFP.

Cleaning performed using probes introduced inside the cartridge and supplied with compressed air was the most efficient of the cleaning methods tested. Cleaning performance did not depend in this case on whether ventilation was kept on or off. Solutions are now being sought to optimise this technique. 
The panel filters tested in this study appeared to be much more efficient regarding the elimination of the particle layer. The greater flexibility of the filter provided by its shape was certainly the reason for the improved performance. Similar results (not given in this report) were obtained with filter bags made of polyester covered with a microporous layer. These tests confirmed that the best cleaning performances were obtained with slackness media, with low elasticity. However, the disadvantage of these filters (panels, pockets, bags) is that they also have small filter surfaces, contrary to the pleated filters used in cartridges and therefore the installations using them are much larger and more expensive.

Regarding the performances in terms of filtration efficiency and downstream particle concentration, the measurements show that they are quite efficient if mass is the reference parameter used, but are much worse if the particle number is considered. Consequently, the air recycling technique cannot be used, under any circumstances, for these particulate pollutants.

\section{References}

[1] A.D. Maynard, Is engineered nanomaterial exposure a myth ?, www.safenano.org (2007).

[2] S. Bicocchi, C. L'hospitalier, Les techniques de dépoussiérage des fumées industrielles, ed. TEC\&DOC, Paris (2002).

[3] H.G. Chong, T.J. Overcamp, Effects of the diffuser and of the particle interception on the penetration of a venture scrubber, Environmental Technology, 17(1) (1996), pp. 107-111.

[4] D. Bémer, R. Régnier, I. Subra, Ultrafine particles emitted by flame and electric arc guns for thermal spraying of metals. Ann. Occup. Hyg. 54(6) (2010), pp. 607-614.

[5] O. Witschger, Les Nanoparticules. Avis d'experts, ed INRS, EDP Sciences Les Ulis (2007).

[6] D. Thomas, P. Penicot, P. Contal, D. Leclerc, J. Vendel, Clogging of fibrous filters by solid aerosol particles, Experimental and modeling study. Chemical Engineering Science 56(11) (2001), pp. 3549-3561.

[7] Guide de ventilation $n^{\circ} 14$ - Décapage, dessablage, dépolissage au jet libre en cabine, ed. INRS 768 (2004).

[8] L.M. Lo, D-R. Chen, D.Y.H. Pui, Experimental study of pleated fabric cartridges in a pulse-jet cleaned dust collector. Powder Tech. 197 (2010), pp. 141-149.

[9] E. Ravert, Precoating new filters for better airflow, longer filter life, Powder and Bulk Engineering (2006).

[10] P.H. McMurry, X. Wang, K. Park, K. Ehara, The relationship between mass and mobility for atmospheric particles: a new technique for measuring particle density. Aerosol Science and Technology, 36, (2002), pp.227-238. 
[11] P. Van Broekhuizen et al., Worplace exposure to nanoparticles and the application of provisional nanoreference values in times of uncertain risks. J. Nanoparticles Research 14 (2012), pp. 2-25.

[12] D. Koch, J. Seville, R. Clift, Dust cake detachment from gas filters. Powder Tech. 86 (1996), pp. 21-29.

[13] R. Dennis, J.E. Wilder, D.L. Harmon, Predicting pressure drop for pulse-jet filters. J. of Air Pollution Control Association, 31(9), (1981), pp.987-992. 


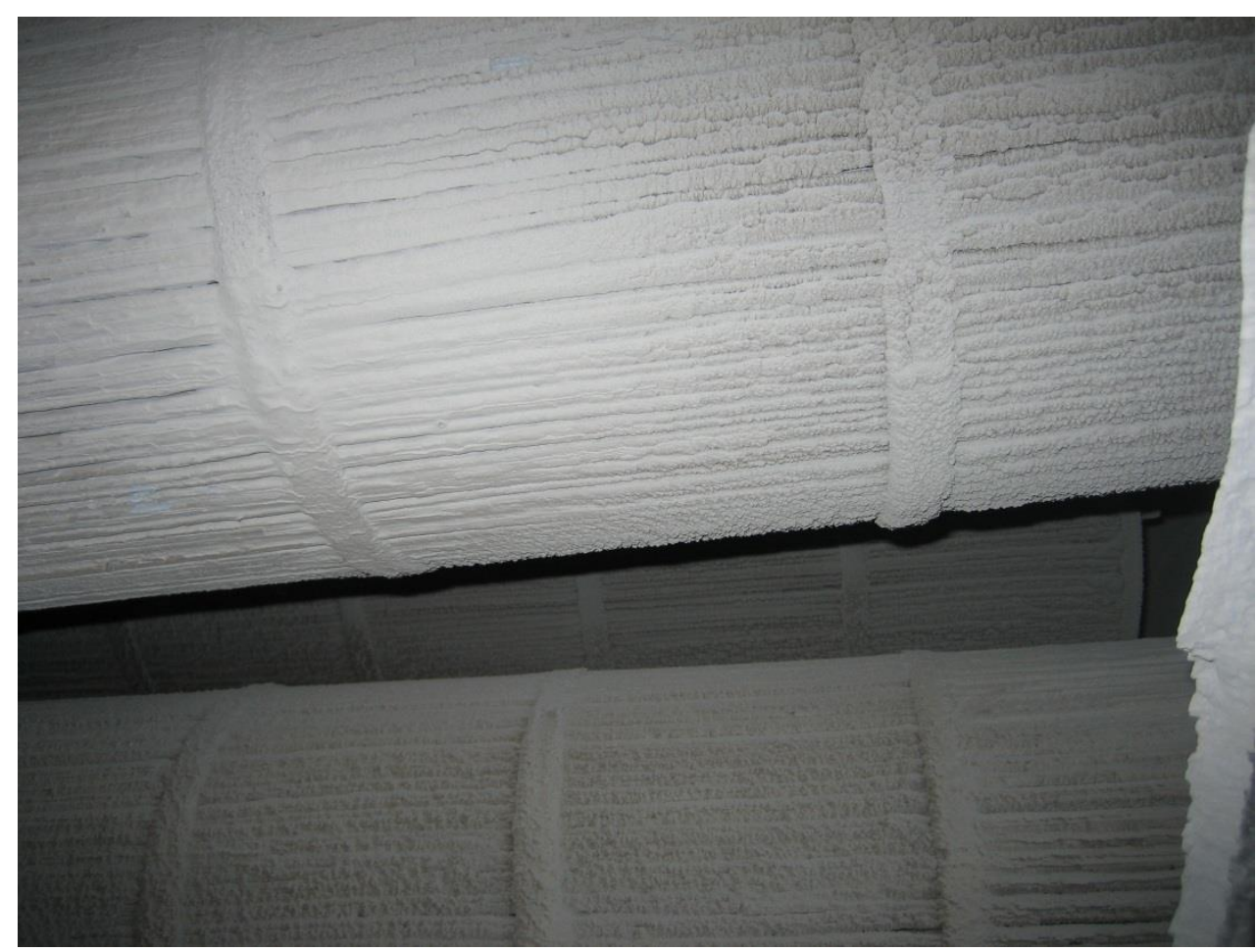

Figure 1. Clogged pleated cartridge filter - dust collector installed on a booth used for thermal spraying with an electric arc gun. 


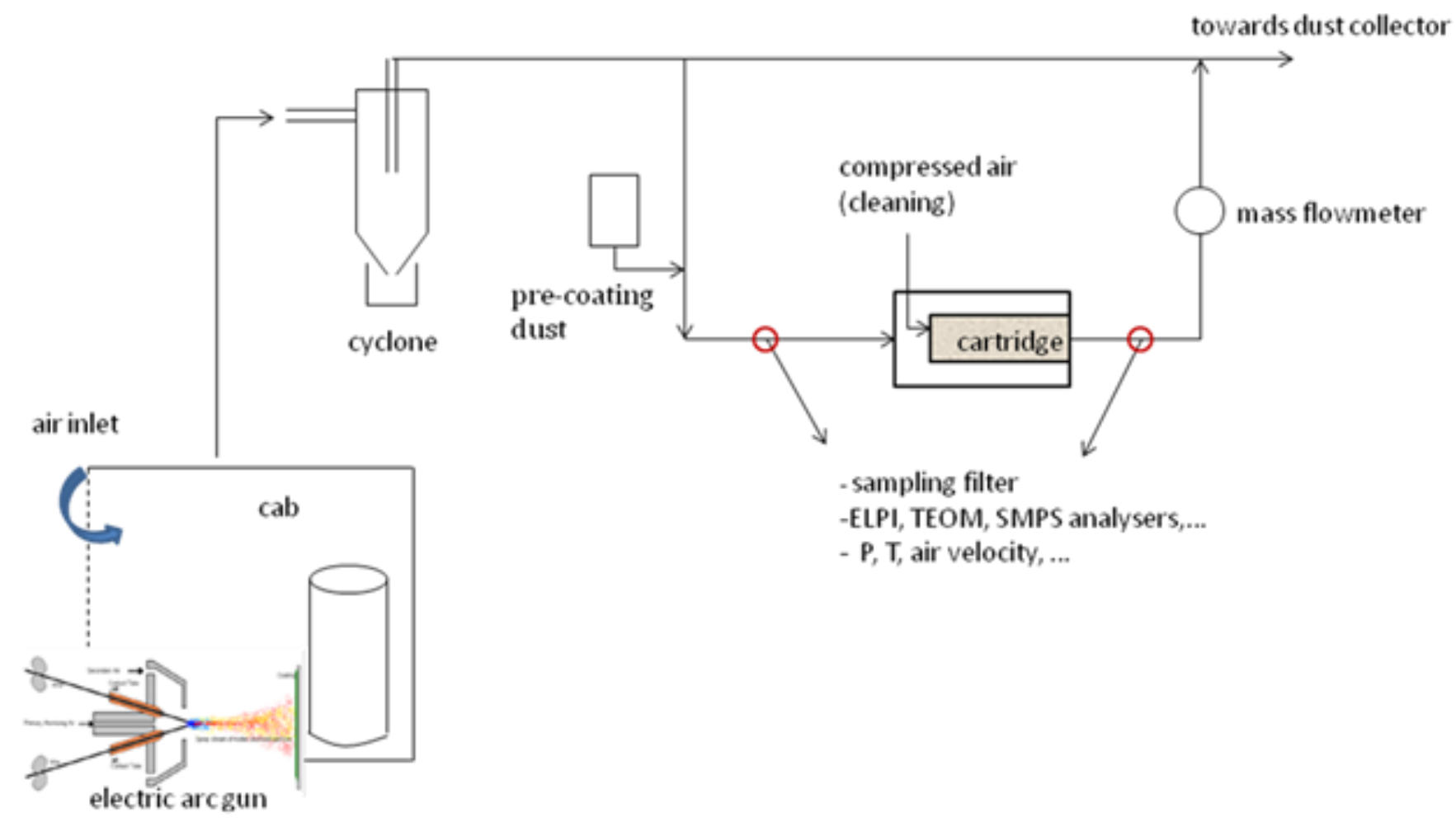

Figure 2. Experimental set-up for measuring the performances of filters subjected to metallic metal spraying fumes (electric arc thermal spraying). 


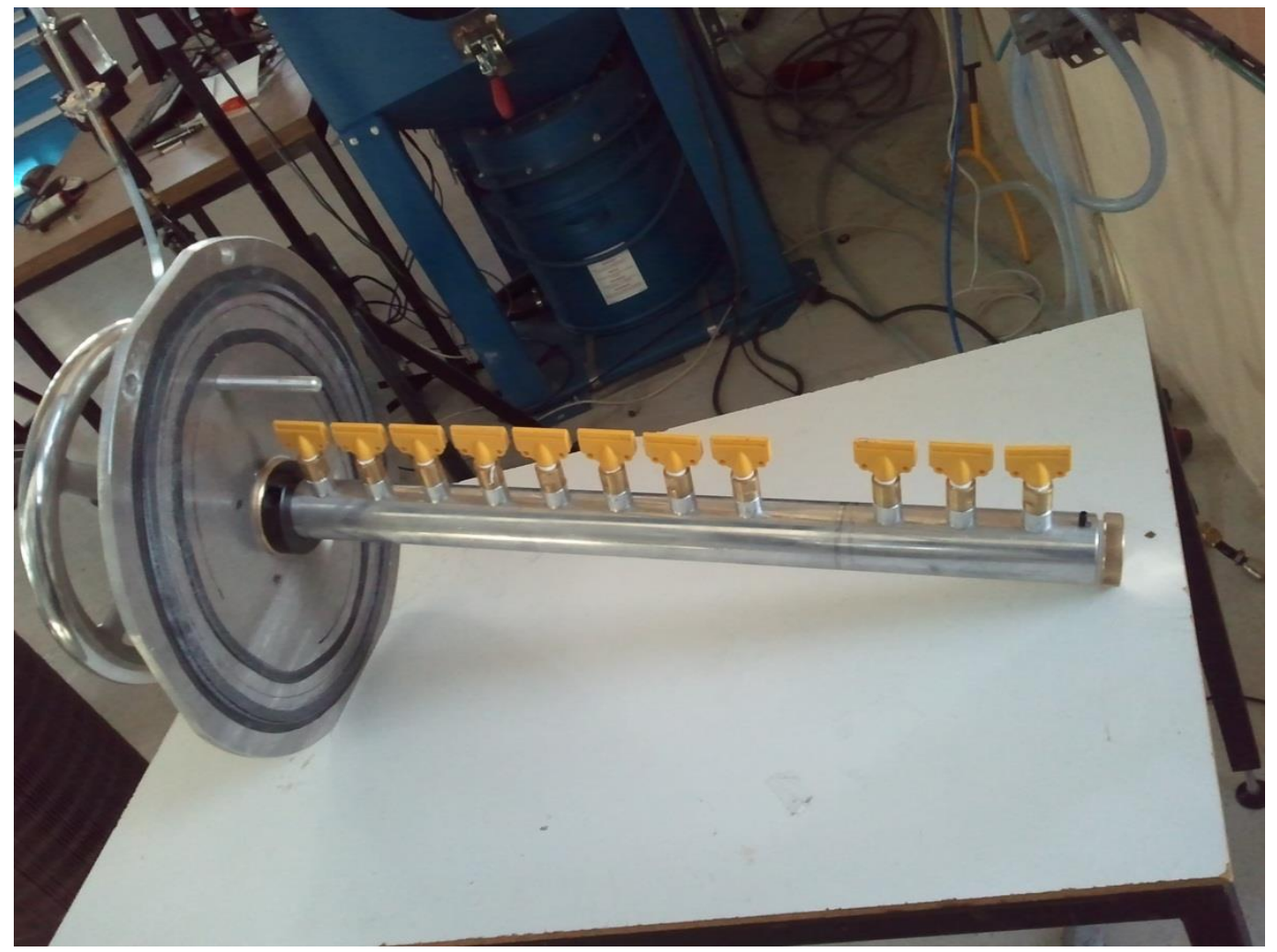

Figure 3. Photograph of the cleaning probe used for filter cartridges by blowing compressed air. 


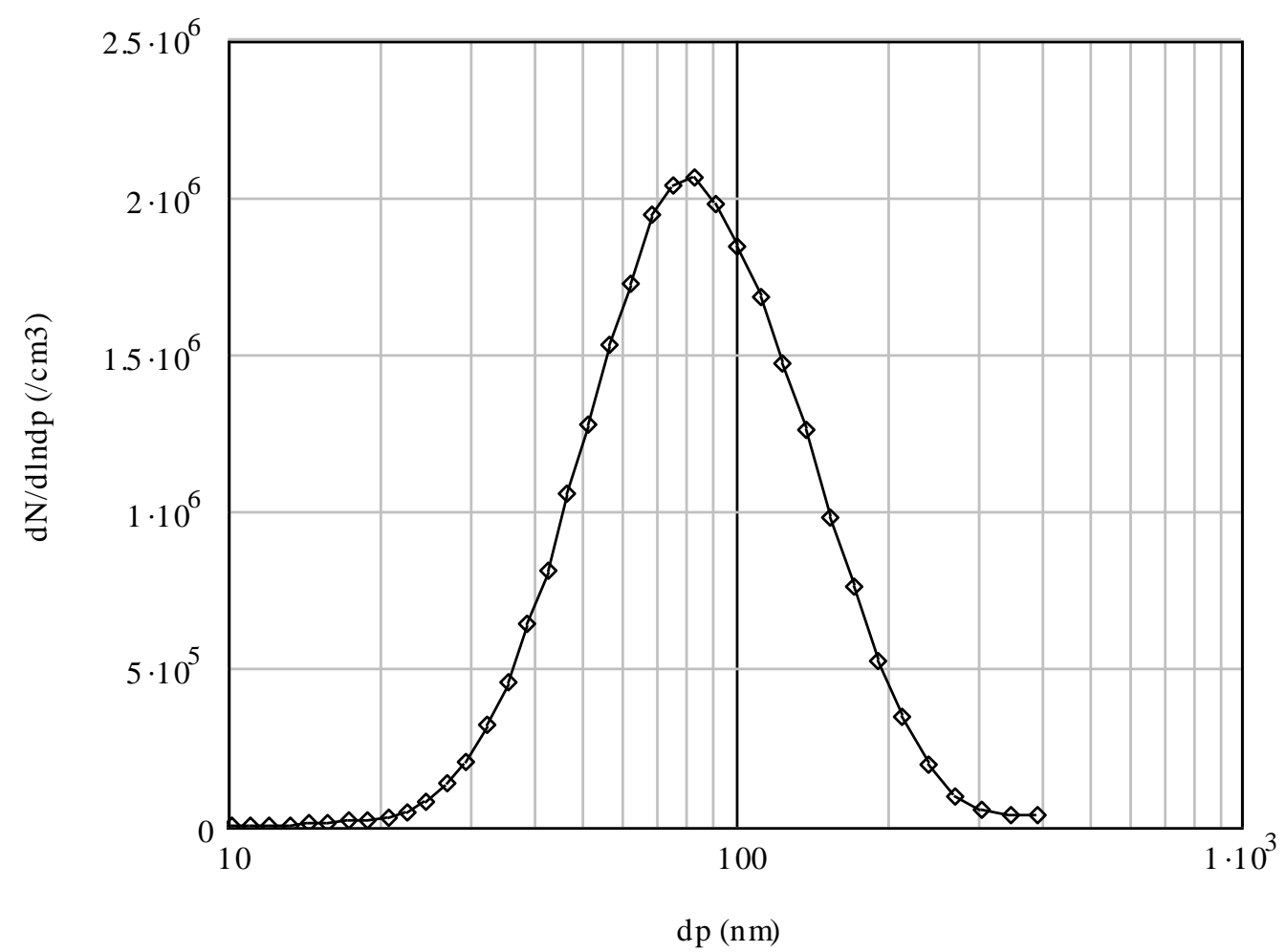

Figure 4. Particle size distribution in number of metallic particles - SMPS measurement dilution of the sample $1 / 100$. 


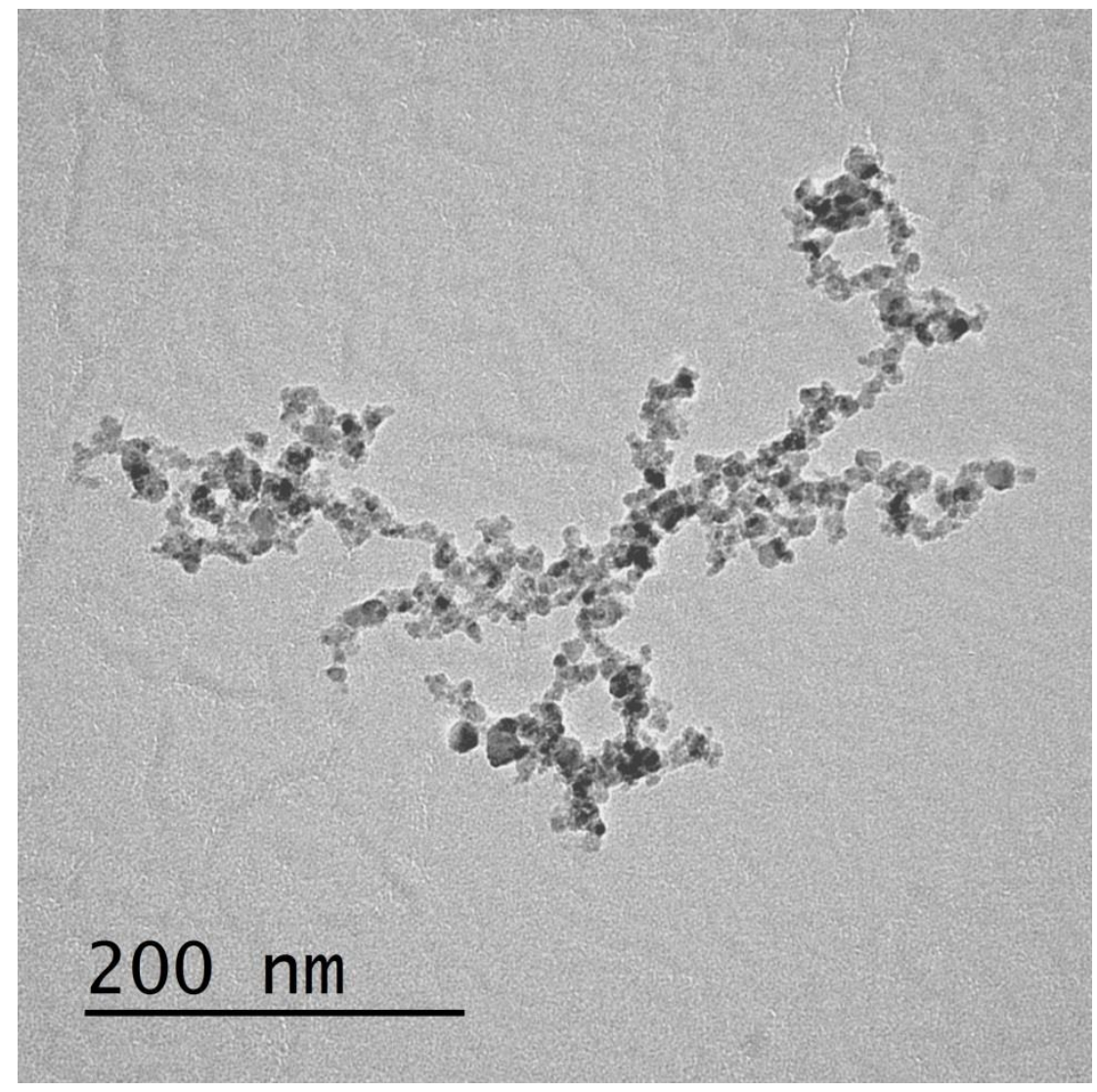

Figure 5. Transmission electron microscope photograph of the metallic particles generated by thermal spraying (electric arc gun). 


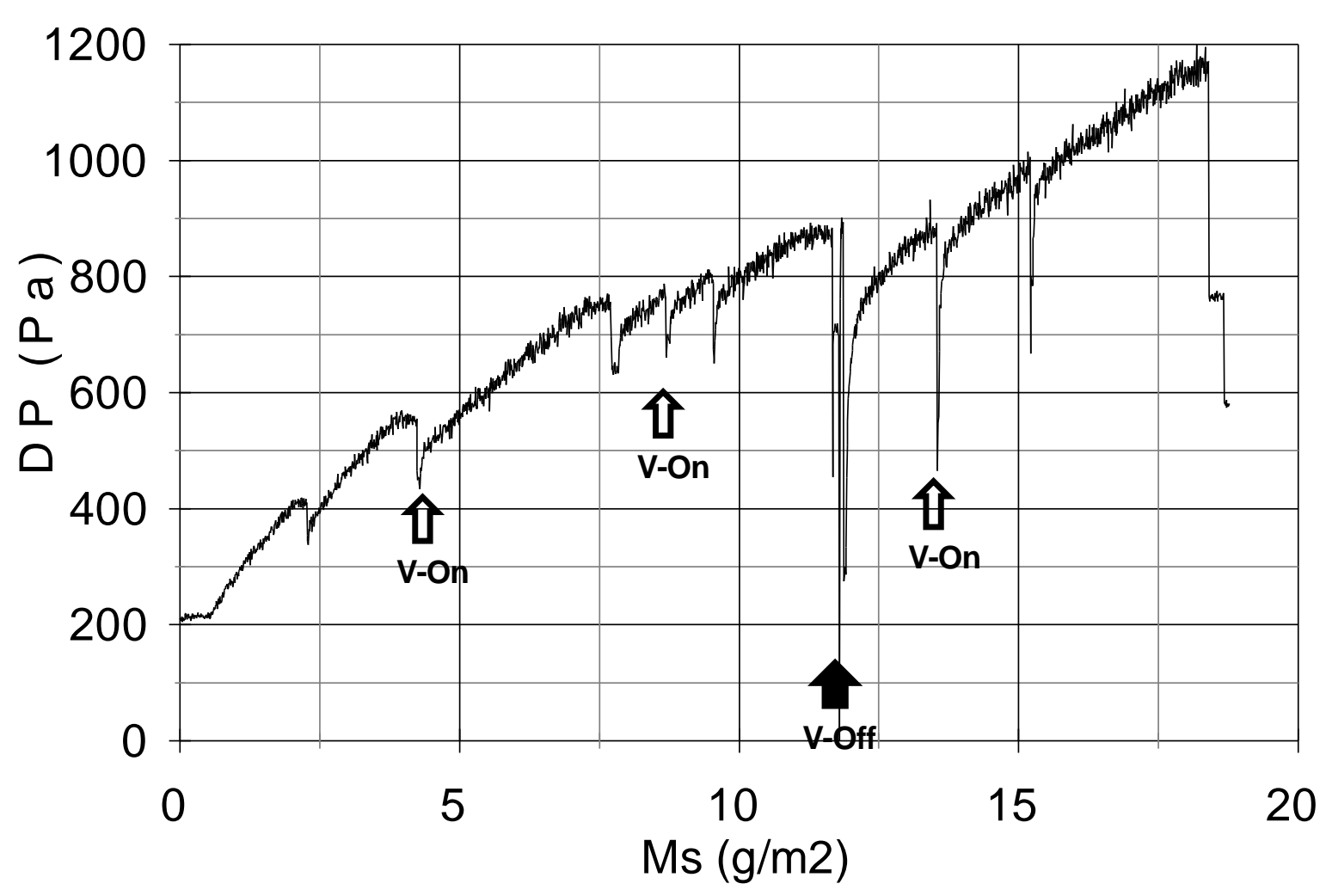

Figure 6. Evolution of pressure drop (cartridge B) during clogging and cleaning (V-on and Voff). Flow of particles collected by the filter equal to $3.4 \mathrm{~g} / \mathrm{m}^{2} / \mathrm{h}$. 


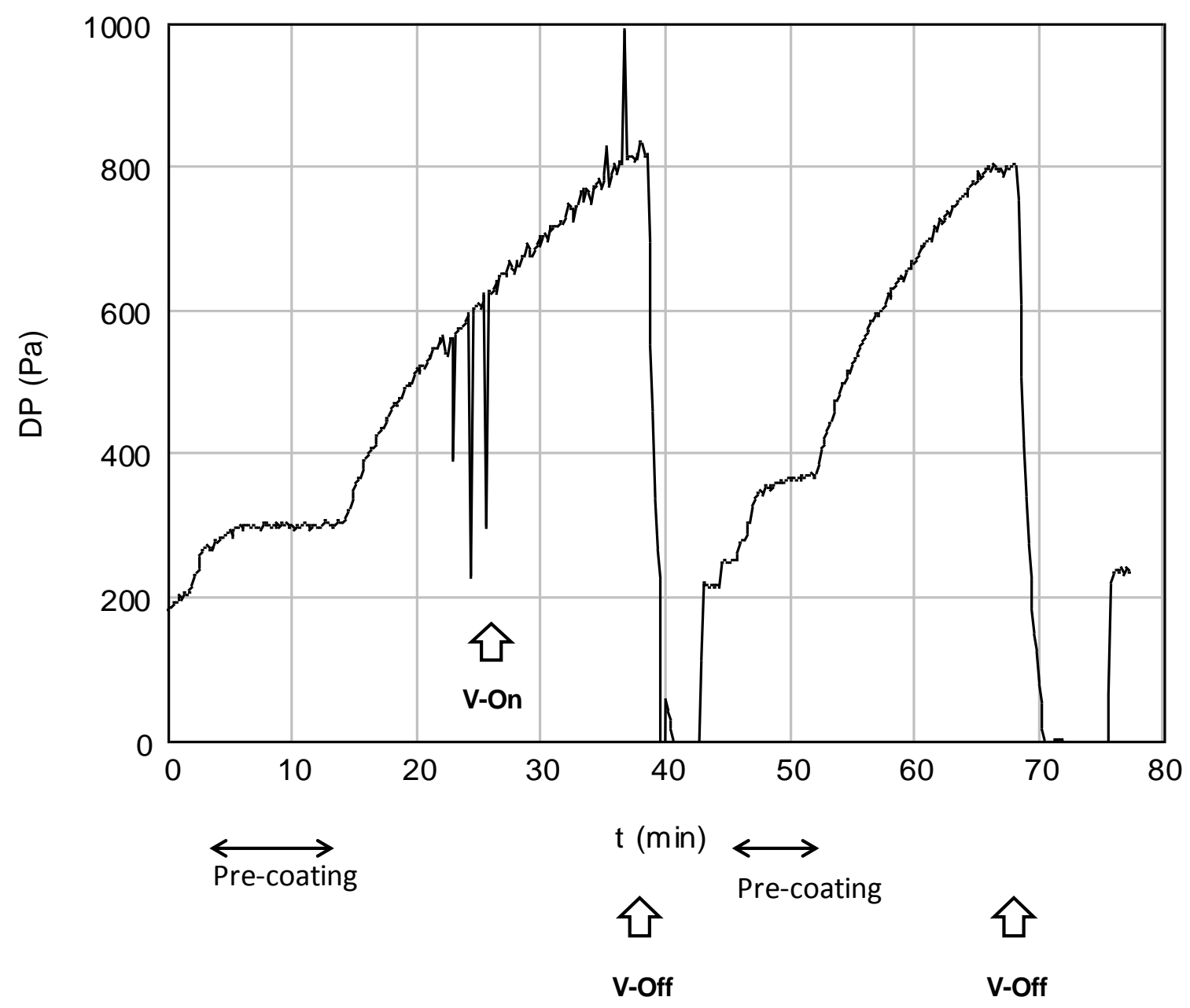

Figure 7. Evolution of pressure drop (cartridge $\mathrm{B}$ ) during clogging and cleaning (V-on and Voff) after pre-coating with limestone dust $3.6 \mu \mathrm{m}$. 


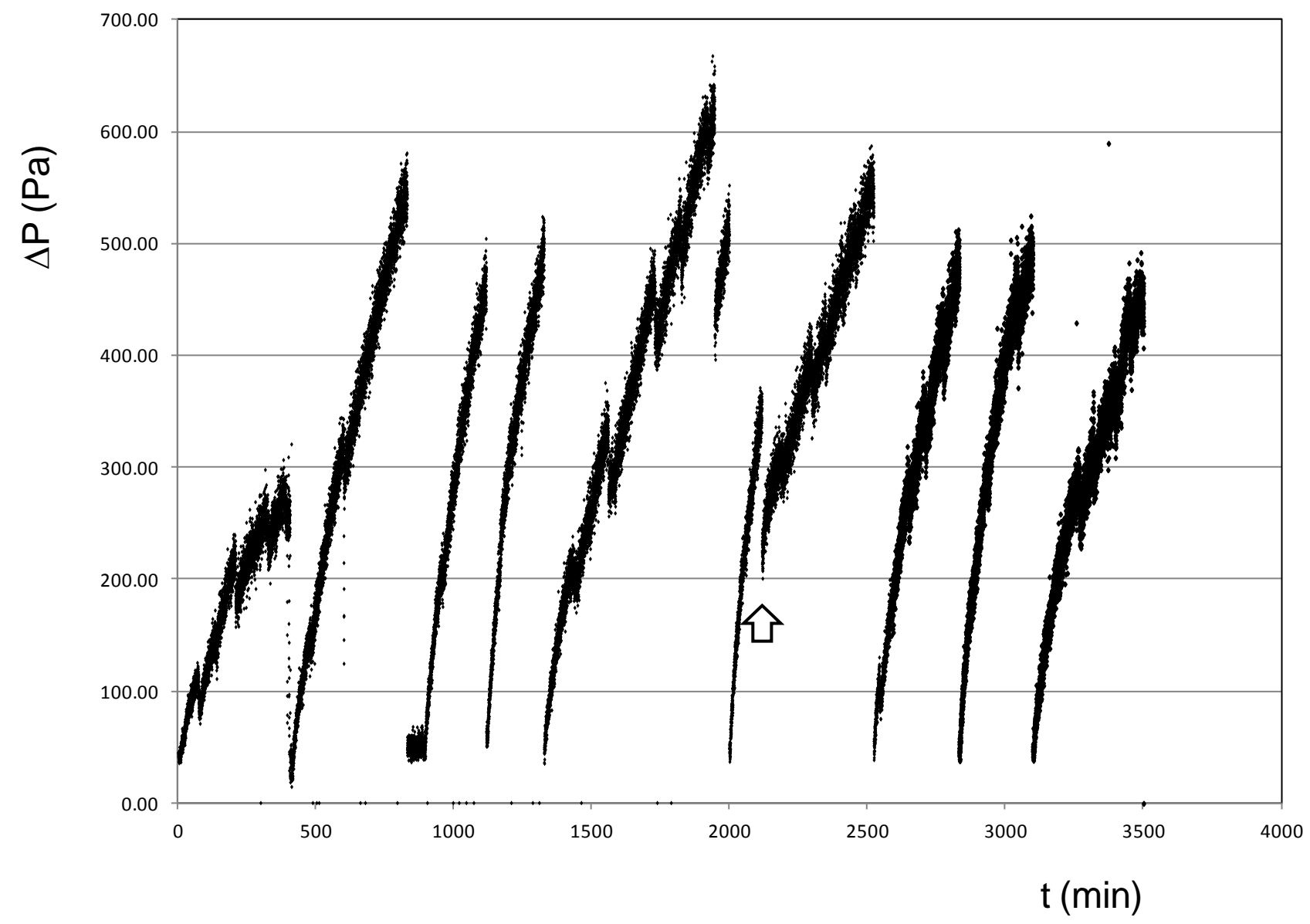

Figure 8. Evolution of the pressure drop of the panel filter during clogging and cleaning cycles. The arrow indicates the interruption of several days. 


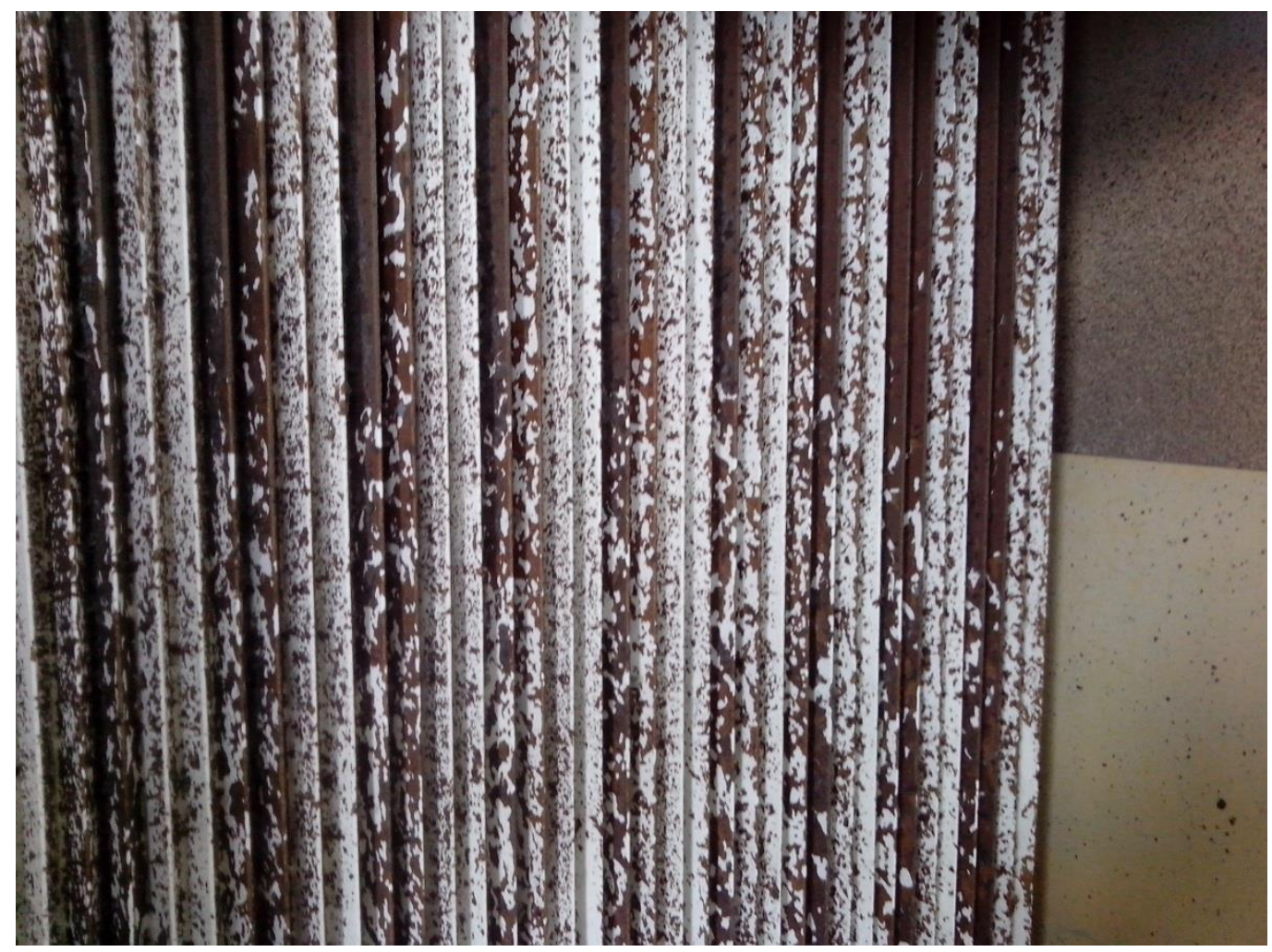

Figure 9. View of the panel filter after cleaning (off-line) showing the partially detached filter cake. 


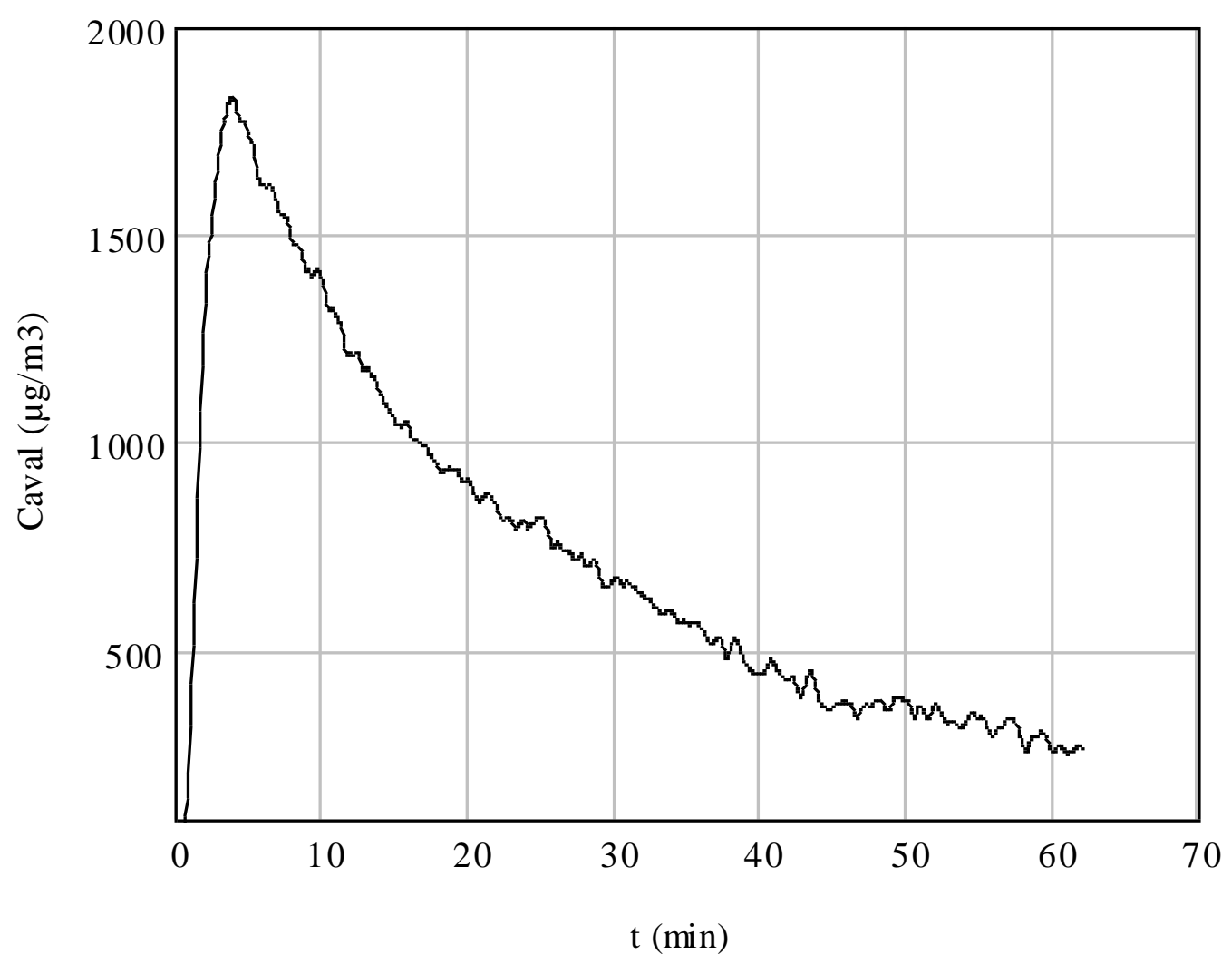

Figure 10. Evolution of the particle mass concentration concentration downstream of the panel filter during clogging -TEOM measurement. 